\title{
Fine-scale population differentiation and gene flow in a terrestrial salamander (Plethodon cinereus) living in continuous habitat
}

\author{
PR Cabe, RB Page ${ }^{1}$, TJ Hanlon, ME Aldrich, L Connors and DM Marsh \\ Department of Biology, Washington and Lee University, Lexington, VA, USA
}

\begin{abstract}
Several recent studies have shown that amphibian populations may exhibit high genetic subdivision in areas with recent fragmentation and urban development. Less is known about the potential for genetic differentiation in continuous habitats. We studied genetic differentiation of red-backed salamanders (Plethodon cinereus) across a 2-km transect through continuous forest in Virginia, USA. Mark-recapture studies suggest very little dispersal for this species, whereas homing experiments and post-Pleistocene range expansion both suggest greater dispersal abilities. We used six microsatellite loci to examine genetic population structure and differentiation between eight subpopulations of redbacked salamanders at distances from $200 \mathrm{~m}$ to $2 \mathrm{~km}$. We also used several methods to extrapolate dispersal frequencies and test for sex-biased dispersal. We found small, but detectable differentiation among populations, even at distances as small as $200 \mathrm{~m}$. Differentiation was closely
\end{abstract}

correlated with distance and both Mantel tests and assignment tests were consistent with an isolation-by-distance model for the population. Extrapolations of intergenerational variance in spatial position $\left(\sigma^{2}<15 \mathrm{~m}^{2}\right)$ and pair-wise dispersal frequencies $(4 \mathrm{Nm}<25$ for plots separated by $300 \mathrm{~m}$ ) both suggest limited gene flow. Additionally, tests for sex-biased dispersal imply that dispersal frequency is similarly low for both sexes. We suggest that these low levels of gene flow and the infrequent dispersal observed in markrecapture studies may be reconciled with homing ability and range expansion if dispersing animals rarely succeed in breeding in saturated habitats, if dispersal is flexible depending on the availability of habitat, or if dispersal frequency varies across the geographic range of red-backed salamanders.

Heredity (2007) 98, 53-60. doi:10.1038/sj.hdy.6800905; published online 27 September 2006

Keywords: red-backed salamander; sex-biased dispersal; gene flow; microsatellites; population structure; Plethodon cinereus

\section{Introduction}

The study of population genetic structure in fragmented environments has become a cornerstone of ecological genetics research. Studies of genetic differentiation in fragmented habitats can determine the extent to which genetic diversity is lost in isolated populations (Avise and Hamrick, 1996; Burns et al., 2004; Funk et al., 2005) and identify the landscape features that restrict dispersal and gene flow (Lampert et al., 2003; Funk et al., 2005). These studies can also identify appropriate management units and determine the extent to which individual subpopulations should be protected as distinct genetic units (Shaffer et al., 2000). Given the accelerating pace of fragmentation across the globe, most large-scale studies of population genetics are de facto studies of habitat fragmentation. As a result, for many organisms we often know less about patterns of genetic differentiation in continuous habitats than in recently fragmented habitats. This is unfortunate, because an understanding of

Correspondence: Dr PR Cabe, Department of Biology, Washington and Lee University, Howe 301, Science Center, Lexington, VA 24450, USA. E-mail: cabep@wlu.edu

${ }^{1}$ Current address: Department of Biology, University of Kentucky, Lexington, KY 40506, USA

Received 21 February 2006; revised 17 July 2006; accepted 26 August 2006; published online 27 September 2006 patterns of genetic structure in continuous habitats is essential for determining how these patterns are altered by fragmentation. In addition, continuous environments provide the appropriate historical backdrop for the evolution of many species.

We chose to study the genetic structure of red-backed salamanders (Plethodon cinereus) in continuous forest habitat in the Blue Ridge Mountains of southwest Virginia. In this species, eggs are laid in clutches in damp microenvironments under rocks or woody debris. As is typical of this genus and many other plethodontid salamanders, direct development of the eggs makes this salamander completely terrestrial in all life stages.

Terrestrial plethodontid salamanders have served as models for the study of behavioral and community ecology (see Petranka (1998) for reviews), and a better understanding of the fine-scale genetic structure of this group in a natural environment could contribute to the understanding of scientists working in both of these disciplines. For example, most behavioral studies of redbacked salamanders ( $P$. cinereus) have used salamanders from a single area in Southwestern Virginia (e.g. Jaeger, 1981; Mathis, 1990, 1991; Gillette, 2003). The potential for local adaptation in behavior and the scale over which observed behaviors are ecologically relevant will depend on the extent of dispersal, gene flow, and genetic differentiation in these animals. Additionally, terrestrial 
salamander populations are increasingly being monitored as an index of forest health (e.g. Amphibian Research and Monitoring Initiative, North American Amphibian Monitoring Program, Partners in Amphibian and Reptile Conservation). An improved understanding of dispersal and gene flow could help determine the appropriate scale for selection of monitoring units.

The extent of dispersal in terrestrial plethodontid salamanders is poorly understood. These salamanders defend cover objects on the forest floor and oviposit at these sites (Mathis, 1989, 1991). Because eggs hatch to small terrestrial juveniles, migration to aquatic sites is not a part of the life-history of these animals. Markrecapture studies consistently show small home ranges $\left(5-25 \mathrm{~m}^{2}\right)$ and very little movement among cover objects (Kleeberger and Werner, 1982; Mathis, 1991; Marvin, 1998), even across multiple years (Gillette, 2003). However, Kleeberger and Werner (1982) showed that adult red-backed salamanders could home successfully at distances up to $90 \mathrm{~m}$ - much larger distances than any observed home range. Additionally, Marsh et al. (2004) found that young adult red-backed salamanders readily colonized experimental plots in open fields with movements on the order of 30 or $40 \mathrm{~m}$. Finally, red-backed salamanders have expanded their distribution considerably since the end of Pleistocene glaciation, and their current distribution goes north to Quebec and Nova Scotia and west to Minnesota and Wisconsin. Assuming a period of range expansion of 18000 years, their rate of expansion is approximately $80 \mathrm{~m}$ /year. Thus, ecological data present somewhat conflicting suggestions about the dispersal abilities of terrestrial salamanders; genetic data may be useful in resolving these contradictions.

A few previous studies have used genetic markers to investigate population structure in red-backed salamanders. Highton and Webster (1976) used protein electrophoresis to document high levels of divergence among populations throughout the range, although their closest populations were $32 \mathrm{~km}$ apart, and most population samples were separated by hundreds of kilometers. Using the same genetic markers, Highton (1977) also showed marked genetic differences on a finer scale (0.5$35 \mathrm{~km}$ ). Gibbs (1998), using RAPD DNA markers, found that the degree of differentiation among samples was affected by recent forest fragmentation. He also found that in continuous forest, genetic divergence was weakly correlated with distance (Gibbs, 1998). Finally, Sites et al. (2004) analyzed mitochondrial DNA and found that redbacked salamanders formed a monophyletic clade with some differentiation across Virginia, West Virginia and Kentucky, USA. Collectively, these studies suggest that red-backed salamander dispersal and gene flow are limited at the scale of kilometers. However, because these studies were on relatively large scales, they cannot resolve whether terrestrial salamanders regularly disperse moderate distances (as suggested by homing and range expansion) or rarely move at all (as suggested by mark-recapture studies).

We used microsatellite markers to examine genetic population structure in red-backed salamanders along a $2 \mathrm{~km}$ transect passing through continuous forest in the Appalachian Mountains of Southwestern Virginia. This transect contained no open habitats, streams, ridges or other likely barriers to dispersal. We asked whether subpopulations separated by a range of distances from $200 \mathrm{~m}$ to $2 \mathrm{~km}$ were locally differentiated despite the lack of dispersal barriers. We also used several methods to extrapolate dispersal rates from these data. Finally, we used patterns of relatedness to determine whether dispersal in red-backed salamanders appears to differ between males and females.

\section{Materials and methods}

\section{Study area}

Our study site is located in Giles County, Virginia, USA in the Jefferson National Forest at approximately $37.41 \mathrm{~N}$ and $-80.50 \mathrm{~W}$. It ranges in elevation from 1150 to $1250 \mathrm{~m}$. The site consists of mixed deciduous hardwood forest and has no obvious heterogeneity in stand age or potential dispersal barriers such as streams and rocky ridges. Some previous disturbance is expected for all forests in Eastern North America. The occurrence of Pitch Pine (Pinus rigida) and Black Locust (Robinia pseudoacacia) at the site suggest that at least some of the area was previously deforested, although not in the past 70-80 years (HM Wilbur, personal communication). In any case, populations of red-backed salamanders are unlikely to disappear completely even when sites are clearcut (Knapp et al., 2003).

At the study site, we established a straight transect $2 \mathrm{~km}$ long with the aid of a GPS receiver. Along the transect, we marked off eight $50 \mathrm{~m}^{2}$ plots with plots separated by either $200 \mathrm{~m}$ or $300 \mathrm{~m}$ (Figure 1). This design was used as a compromise between the need for a range of pair-wise distances between plots and the need for replicates at each distance.

\section{Collection and laboratory methodology}

We collected 48 red-backed salamanders on each plot between 24 April and 6 August 2003. Salamanders were found by searching under naturally occurring cover objects, such as rocks and logs, and by sifting through the

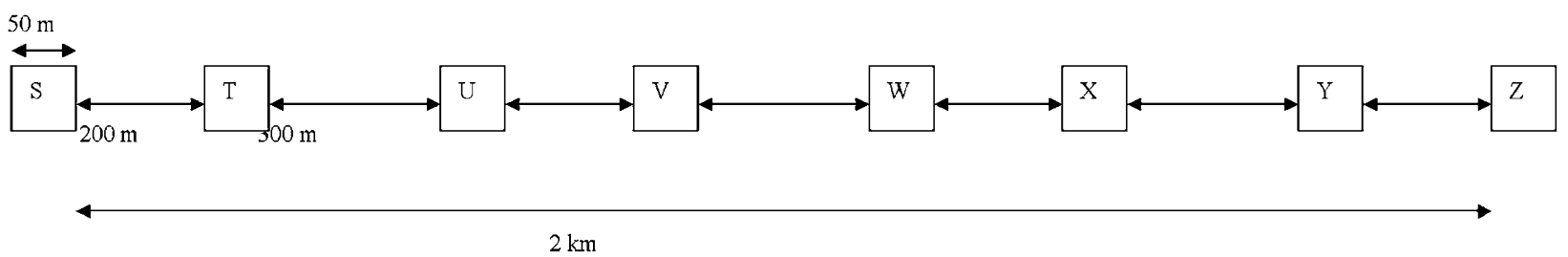

Figure 1 Diagram of study site. Forty-eight samples were collected from each of the eight plots labeled S through Z. 
leaf litter. For all but one plot, all salamanders were collected on a single day to avoid recapturing individuals. We determined the sex of as many individuals as possible in the field by checking for the presence of testes as described by Gillette and Peterson (2001). Juvenile salamanders were not sexed. From each captured salamander, we removed approximately $1 \mathrm{~cm}$ of tail tissue with forceps and placed it in a sterile $1.5 \mathrm{ml}$ microtube containing collection buffer $(10 \mathrm{mM}$ Tris, $10 \mathrm{~mm}$ ethylene diamine tetra acetic acid, $\mathrm{pH} 8$ ). Following tissue collection, salamanders were released at their points of capture. We placed samples on ice during transport to the laboratory, and performed DNA extraction within $24 \mathrm{~h}$. Genomic DNA was extracted using the reagents and suggested protocols from the Promega Wizard Genomic DNA Purification Kit. We ground tissue samples in $500 \mu \mathrm{l}$ of nuclei lysis solution, incubated at $65^{\circ} \mathrm{C}$ for $20-30 \mathrm{~min}$, treated with RNase at $37^{\circ} \mathrm{C}$ for $25 \mathrm{~min}$, treated with $170 \mu$ l of protein precipitation solution, and centrifuged. The supernatant was decanted and the DNA was precipitated using isopropanol. The DNA pellet was then washed with $70 \%$ ethanol, dried and rehydrated with TE $(10 \mathrm{mM}$ Tris, $1 \mathrm{mM}$ ethylene diamine tetra acetic acid, $\mathrm{pH}$ 8.0). DNA samples were stored in a freezer and dilutions of this stock (1:4 or 1:9) were used as templates for polymerase chain reaction (PCR).

We amplified five microsatellite loci (PcI16, PcLX16, PcLX23, PcJX06 and PcFX08) following the protocols detailed in Connors and Cabe (2003). Although the original protocols specified multiplex reactions, some loci were amplified independently to increase yields. One additional locus, PcXD23 (primers HEX/GCAAAA CAGCAACAAGACAAC, AACCTTGATGTTTGGCAAGG; Genbank accession number AY151376) was amplified using similar protocols (Cabe, unpublished).

After verifying the success of our PCR via agarose gel electrophoresis, PCR products were shipped to the Advanced Genetics Analysis Center at The University of Minnesota where they were sized using ABI 377 or 3100 DNA sequencers and GENESCAN (Applied Biosystems, Foster City, California, USA) software. Genotypes were determined in our laboratory using GENOTYPER (Applied Biosystems, Foster City, California, USA) software. We manually inspected each allele call, and manually binned alleles.

\section{Data analysis}

We used microsatellite analyzer (MSA) to obtain observed and expected heterozygosities, allele counts and size ranges (Dieringer and Schlötterer, 2003). We then used GenePop, Version 3.4 (Raymond and Rousset, 1995) to test each locus for Hardy-Weinberg $(\mathrm{H}-\mathrm{W})$ proportions. The probabilities from these tests were adjusted for an experiment-wise probability of 0.05 using the sequential Bonferroni correction suggested by Weir (1990). We tested for linkage disequilibrium for each pair of loci in the same manner. We followed the method of Garza and Williamson (2001) to test for recent reductions in the population size in order to gauge recent demographic history at our site.

We used a variety of approaches to investigate population structure and gene flow. First, we used a Bayesian approach to conduct clustering analyses over several different hierarchical levels. We also used a likelihood-based assignment approach. Finally, we used a traditional population differentiation approach based on $F_{\mathrm{ST}}$ analysis. This latter approach has known limitations for microsatellite data (Hedrick 1999, 2005) but allows comparison with many other published studies and its properties and limitations are well established (Neigel, 2002).

We used Bayesian Analysis of Population Structure (BAPS), Version 4.13 software (Corander et al, 2003; 2004) to conduct hierarchical clustering analyses. These analyses included: (1) clustering individuals independently of the location at which they were sampled, (2) clustering plots and (3) clustering individuals according to an admixture model that accounts for the known origin (i.e., plot) of each individual. The individual clustering was performed by running 10 replicates for each of the following upper bound values of $k: 20,15,10$ and 5 . The plot clustering was performed by running 10 replicates for each of the following upper bound values of $k: 8,4$ and 2 . The admixture clustering was performed using a minimum population size of 10 individuals, 100 iterations, 200 reference individuals from each population and 20 iterations for the reference individuals.

We also used Arlequin Version 2.000 (Schneider et al., 2000) to assess our ability to assign the genotypes of individuals to the plots from which they came. This procedure determines the log-likelihood of observing each multilocus genotype in each of a series of plots. Log-likelihoods are calculated by using the allelic frequencies from the observed data for each plot in the series. The 'global individual likelihood' of an individual coming from a given plot is the product of the likelihood of that individual coming from this plot for each locus of the individual's genotype.

In addition, we used Arlequin Version 2.000 (Schneider et al., 2000) to calculate pair-wise estimates of $F_{\mathrm{ST}}$ between all pairs of plots. The significance of these estimators were assessed using a non-parametric data permutation approach (Excoffier et al., 1992; Schneider et al., 2000). We chose to calculate $F_{\mathrm{ST}}$ values rather than $R_{\mathrm{ST}}$ values because of the better performance of $F_{\mathrm{ST}}$ estimates when divergence among samples is expected to be low (Balloux and Goudet, 2002).

The relationship between genetic and geographic distance was investigated with a Mantel test performed with ZT software (Bonnet and Van de Peer, 2002). Using this procedure, we examined the correlation between the pair-wise $F_{\mathrm{ST}}$ distance matrix and the geographic distance matrix.

Estimates of gene flow and genetic neighborhood size ( $4 \mathrm{Nm}$ or $4 \mathrm{D} \partial \sigma^{2}$, respectively) were obtained via two methods. First, we estimated $4 \mathrm{Nm}$ using Bayesian estimation in Migrate, Version 2.1.3 (Beerli and Felsenstein, 1999, 2001; Beerli, 2004，2006) following the recommendations of Beerli (2004): (1) we did an initial run on our data set using $F_{\mathrm{ST}}$ to find the start parameters and (2) we used the output of the initial run as the start parameters of our second run. Because there were only minor differences between the outputs from the first run and the second run, we stopped after the second run, and present output from the second run in the results below. Second, we used Rousset's (1997) methodology for estimating these parameters for two-dimensional 
habitats. This procedure regresses $F_{\mathrm{ST}} /\left(1-F_{\mathrm{ST}}\right)$ on the natural logarithm of distance.

Both of these methods for estimating migration rates may have important limitations with respect to our data set. The $F_{\mathrm{ST}}$-based approach is limited because of the numerous theoretical assumptions that must be made in order to equate $F_{\mathrm{ST}}$ and gene flow (see Beerli and Felsenstein, 2001; Neigel, 2002). The Bayesian approach is limited by the practical problems associated with estimating migration rates when there are several samples, several loci and a large number of individuals (Neigel, 2002; Beerli, 2006). In addition, Migrate estimates gene flow parameters based on direct dispersal between populations, whereas our populations are embedded within a continuous gene pool. Despite these limitations, we used both methods in the hope that broad, qualitative inferences about dispersal would be consistent across methods.

Finally, we examined the data for evidence of sexbiased dispersal. Within each plot, we calculated a relatedness coefficient, $R$ (Queller and Goodnight, 1989) for each pair of individuals of the same sex, and then compared mean $R$-values for each sex using a $T$-test paired by plot. In general, the sex that disperses less would be expected to show higher average relatedness (Queller and Goodnight, 1989). Statistical significance for this test was determined by 1000 random permutations of genotypes among individuals. In addition, we used assignment indices in $F_{\mathrm{ST}}$ at, Version 2.9.3.2 (Goudet, 2001) as an alternative approach for investigating sexbiased dispersal. This method involves comparing the means of each sex's corrected assignment index (AIc) (Goudet, 2001). The assignment index is based on the probability of a genotype being found in a given sample and is 'corrected' (AIc) for differing levels of allelic diversity among samples (Goudet, 2001). The sex with the higher AIc would be expected to disperse less, and we tested the significance of differences in AIc values between sexes with 10000 permutations of genotypes.

\section{Results}

\section{Summary statistics}

All loci within each sample plot were in $\mathrm{H}-\mathrm{W}$ proportions with a single exception, locus PcLX16 in plot S, which had an excess of heterozygotes. However, deviations from $\mathrm{H}-\mathrm{W}$ of this locus in other sample plots did not approach statistical significance. There was also no evidence for genotypic disequilibrium. Descriptive statistics for the entire study site are given in Table 1 and plot-specific statistics are provided in Table 2.

\section{Recent demographic history}

Although our study site consists of continuous woodland habitat, past logging events could have decimated or locally extirpated red-backed salamanders. Recent $(<100$ generation) reductions in populations are expected to change $M$, the ratio of the number of alleles per locus to the range of allele sizes measured in repeat number (Garza and Williamson, 2001). Owing to low population differentiation, all samples were pooled. Garza and Williamson (2001) encourage caution about data that include odd-sized alleles (not consistent with integer multiples of the repeat motif); nearly every locus in this study had at least few examples of these. For one locus (PcI16), odd-sized alleles were common enough for us to forego calculation of $M$; for the remainder, these odd-sized alleles were omitted from the analysis, which may produce a conservative bias in the estimate of $M$. For these five loci, values of $M$ averaged 0.78 (range 0.6-0.94). These values are not indicative of populations with recent severe reductions, which typically show mean $M$ values below about 0.70 . One caveat to this interpretation is the ability of mutation and/or migration to erase the bottleneck signature. Garza and Williamson's (2001) model explicitly includes mutation, but allows no adjustments for migration. In our case, this might have occurred if the population in our study site had been greatly decreased, and then augmented via migration from other, undisturbed sites. As there are no old-growth areas near our study site, we find this explanation unlikely. Thus, this analysis is consistent with a popula-

Table 1 Genetic diversity of pooled samples, including observed and expected heterozygosities, and site-wide fixation indices $\left(F_{\mathrm{ST}}\right)$ for each locus

\begin{tabular}{lcccccc}
\hline Locus & $\mathrm{N}$ & Alleles & $\mathrm{H}_{O}$ & $\mathrm{H}_{E}$ & $\mathrm{~F}_{S T}$ & $\mathrm{M}$ \\
\hline PcI16 & 368 & 10 & 0.32 & 0.30 & 0.001 & $\mathrm{NA}$ \\
PcLX16 & 384 & 10 & 0.72 & 0.73 & $0.031^{* * *}$ & 0.75 \\
PcLX23 & 381 & 23 & 0.47 & 0.43 & $0.053^{* * *}$ & 0.94 \\
PcJX06 & 376 & 19 & 0.61 & 0.62 & $0.020^{* * *}$ & 0.69 \\
PcFX08 & 369 & 18 & 0.85 & 0.82 & $0.010^{* *}$ & 0.93 \\
PcDX23 & 319 & 10 & 0.78 & 0.82 & $0.007^{*}$ & 0.6 \\
\hline
\end{tabular}

${ }^{*}<0.05,{ }^{* *}<0.005$ and ${ }^{* * *}<0.0005$.

$M$ measures the ratio of the number of alleles per locus to the range in size (see the text).

Table 2 Intraplot demographic data and loci descriptors

\begin{tabular}{|c|c|c|c|c|c|c|}
\hline Plot & Males & Females & Unsexed & $\mathrm{A} / \mathrm{L} \pm$ s.e. & $\mathrm{H}_{E} \pm$ s.e. & $\mathrm{H}_{O} \pm$ s.e. \\
\hline S & 17 & 17 & 14 & $7.333 \pm 0.843$ & $0.611 \pm 0.080$ & $0.607 \pm 0.091$ \\
\hline $\mathrm{T}$ & 18 & 14 & 16 & $5.600 \pm 1.661$ & $0.512 \pm 0.129$ & $0.529 \pm 0.127$ \\
\hline $\mathrm{U}$ & 25 & 13 & 10 & $8.200 \pm 1.960$ & $0.636 \pm 0.078$ & $0.638 \pm 0.092$ \\
\hline $\mathrm{V}$ & 17 & 21 & 10 & $8.600 \pm 1.568$ & $0.601 \pm 0.087$ & $0.630 \pm 0.097$ \\
\hline W & 21 & 20 & 7 & $8.400 \pm 1.833$ & $0.602 \pm 0.090$ & $0.588 \pm 0.097$ \\
\hline$X$ & 20 & 13 & 15 & $8.800 \pm 1.497$ & $0.627 \pm 0.078$ & $0.617 \pm 0.091$ \\
\hline$Y$ & 13 & 11 & 24 & $9.400 \pm 1.364$ & $0.609 \pm 0.088$ & $0.652 \pm 0.109$ \\
\hline $\mathrm{Z}$ & 0 & 0 & 48 & $9.167 \pm 1.352$ & $0.704 \pm 0.074$ & $0.706 \pm 0.070$ \\
\hline
\end{tabular}

$A / L \pm$ s.e. $=$ average number of alleles per locus \pm standard error; $H_{\mathrm{E}} \pm$ s.e. $=$ average expected heterozygosity \pm standard error; $H_{\mathrm{O}} \pm$ s.e. $=$ average observed heterozygosity \pm standard error. 
tion history that includes no drastic bottlenecks, although we cannot rule out past population declines.

\section{Population structure}

Based on our log-likelihood assignment tests, our ability to correctly assign individuals to the plots from which they came ranged from 0.82 for plots separated by $200 \mathrm{~m}$ to 0.94 for plots separated by $2 \mathrm{~km}$ (Figure 2). The strong positive relationship between correct assignment probability and distance is consistent with the results of the Mantel test (see below), and supports an isolation-bydistance model of population structure.

The individual level analysis performed in BAPS recovered 15 clusters (minimum $=1$ individual, maximum $=45$ individuals, median $=33$ individuals) with a posterior probability of 1.00 . The output from this analysis allowed us to assign 'dominance' to plots within a cluster based on which plot within a given cluster was represented by the most individuals. Because one cluster contained a single individual and another cluster contained two individuals from different plots, 'dominance' could not be established for these plots and they are excluded from the subsequent summarization. The minimum proportion of a cluster consisting of individuals from a dominant plot was 0.18 (cluster size $=44$ individuals) and the maximum was 0.57 (cluster size $=37$ ). The median proportion of a cluster consisting of individuals from a dominant plot was 0.29 (minimum cluster $\operatorname{size}=4$ and maximum cluster size $=45)$. We extended this approach to consider the adjacent plots to the dominant plot (either 200 or $300 \mathrm{~m}$ apart depending on the plot) as part of the proportion. Excluding the plots at both ends of the transects (S and Z, Figure 1), adding the adjacent plots flanking both sides of the dominant plot resulted in a median of 0.52 (minimum $=0.40$, maximum $=0.77$ ) of the individuals coming from the dominant plot or the two adjacent plots (minimum cluster size $=4$, maximum cluster size $=43$, median cluster size $=36$ ).

The analysis in which we used BAPS to cluster whole plots suggested that the plots were homogenous (i.e., optimal $k=1$ ). However, the clustering that was performed using an admixture model that incorporated the plot of origin for each sample recovered seven groups, one fewer than the number of plots. As is shown in Figure 2, there is relatively high concordance between

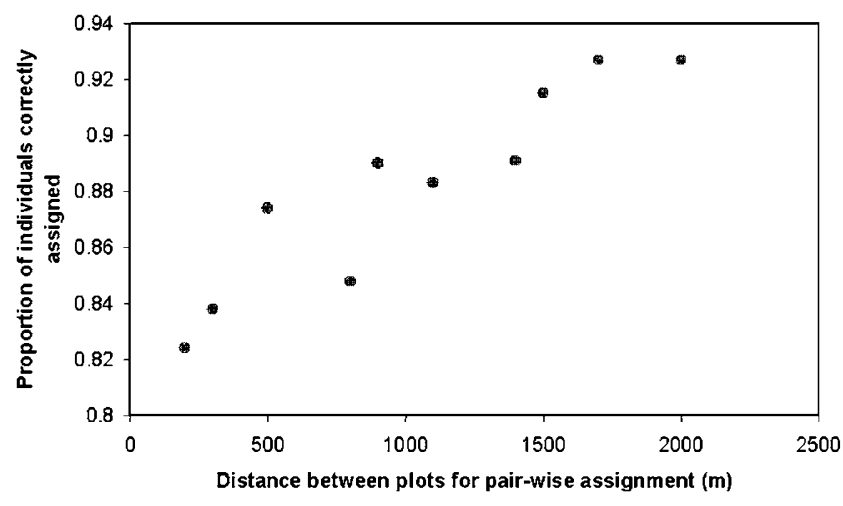

Figure 2 Results of assignment tests for paired plots at each distance. plot of origin and the group from which the largest proportion of an individual's genome is estimated to be derived. Plot $\mathrm{Z}$ was not recovered as a group, and its individuals were nearly evenly distributed in the remaining seven groups. Taken together, these Bayesian analyses suggest that our transect shows slight but detectable fine-scale population structure and isolationby-distance (Figure 3).

Tests for genetic structure yielded a small but highly significant site-wide fixation index $\left(F_{\mathrm{ST}}=0.019\right.$, $P<0.00001$ ). More surprisingly, the majority (23 of 28) of the pair-wise plot comparisons also yielded significant $F_{\mathrm{ST}}$ values (Table 3 ). The estimated probabilities imply strong significance with 18 of the comparisons yielding $P<0.001$. Three of these nonsignificant comparisons were between adjacent plots and a single plot separated the remaining two. The Mantel test showed a strong positive correlation between $F_{\mathrm{ST}}$ and geographic distance $(r=0.723, P=0.001$, Figure 4$)$.

Our estimates of gene flow are consistent with isolation-by-distance. The mean Bayesian estimates of $4 \mathrm{Nm}$ ranged from 24.5 for pairs of plots that were $300 \mathrm{~m}$ apart to 13.3 for plots $1800 \mathrm{~km}$ apart. This extrapolates to roughly six migrants exchanged per generation at $300 \mathrm{~m}$ and three per generation at $1800 \mathrm{~m}$, although these estimates are only meaningful as relative measures. Over

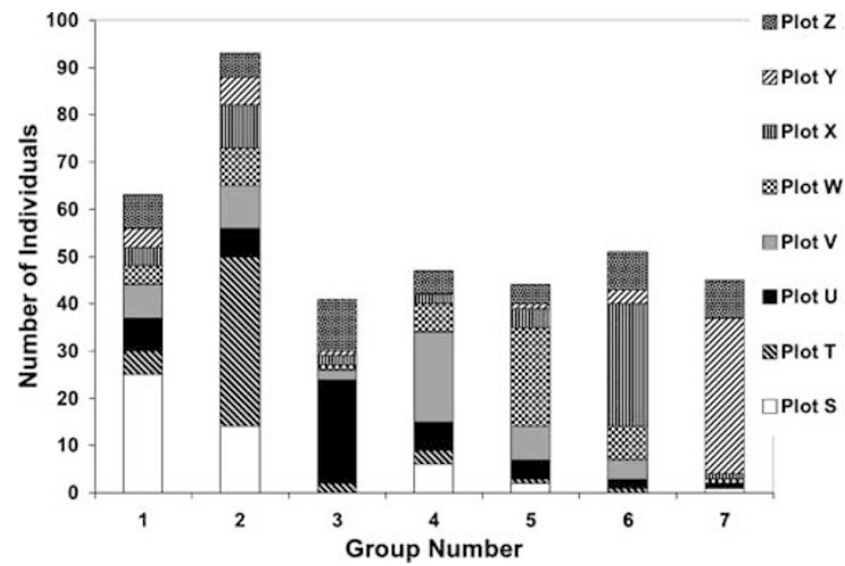

Figure 3 Visualization of the results of the admixture clustering that was performed in BAPS. Each individual is assigned to one of the seven groups based on which group the largest proportion of the individual's genome was estimated to be derived from. Note that a plurality of each group corresponds to a single plot.

Table 3 Pair-wise $F_{\mathrm{ST}}$ values and significance (above diagonal, $\left.{ }^{*}<0.05,{ }^{* *}<0.005,{ }^{* * *}<0.0005\right)$ probability estimates based on 10100 haplotype permutations

\begin{tabular}{lcccccccc}
\hline & $S$ & $T$ & $U$ & $V$ & $W$ & $X$ & $Y$ & $Z$ \\
\hline $\mathrm{S}$ & & $* *$ & $\mathrm{NS}$ & $*$ & $* *$ & $* * *$ & $* * *$ & $* * * *$ \\
$\mathrm{~T}$ & 0.015 & & $* * *$ & $* * *$ & $* * *$ & $* * *$ & $* * *$ & $* * *$ \\
$\mathrm{U}$ & 0.002 & 0.029 & & $\mathrm{NS}$ & $*$ & $*$ & $* * *$ & $* *$ \\
$\mathrm{~V}$ & 0.010 & 0.026 & 0.005 & & $\mathrm{NS}$ & $\mathrm{NS}$ & $* * *$ & $* * *$ \\
$\mathrm{~W}$ & 0.013 & 0.035 & 0.008 & -0.003 & & $\mathrm{NS}$ & $* * *$ & $* *$ \\
$\mathrm{X}$ & 0.017 & 0.028 & 0.010 & 0.003 & 0 & & $* * *$ & $* * *$ \\
$\mathrm{Y}$ & 0.029 & 0.043 & 0.026 & 0.023 & 0.028 & 0.019 & & $* * *$ \\
$\mathrm{Z}$ & 0.016 & 0.061 & 0.011 & 0.017 & 0.014 & 0.019 & 0.033 & \\
\hline
\end{tabular}

NS, nonsignificant. 
58

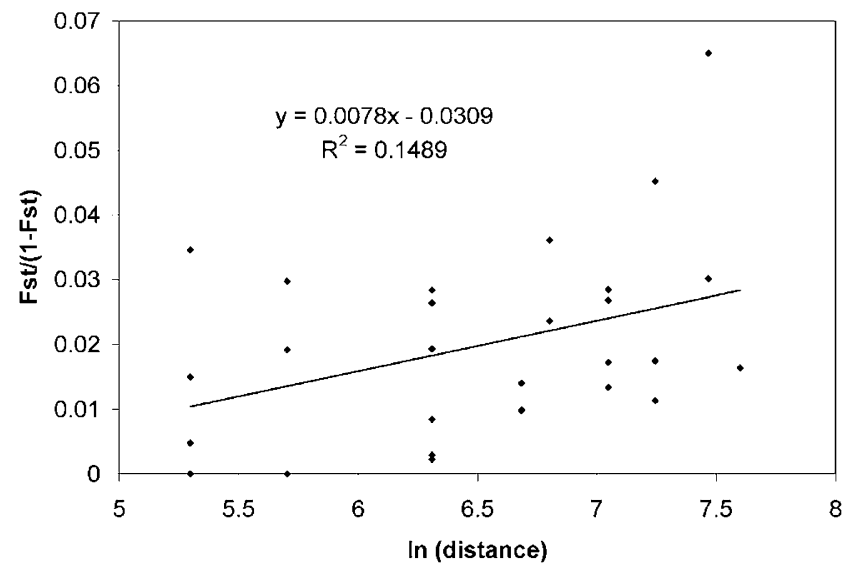

Figure 4 Relationship between $F_{\mathrm{ST}}$ and pair-wise distance between plots along the transect, following Rousset (1997) (see the text).

the range of distances, there was a strong negative correlation between linear distance and estimates for migration rates between plots $\left(r_{\mathrm{p}}=-0.81\right)$.

The regression of $F_{\mathrm{ST}} /\left(1-F_{\mathrm{ST}}\right)$ on $\ln$ (distance) yielded a slope of 0.008 . This slope was used to estimate $1 /\left(4 \mathrm{D} \partial \sigma^{2}\right)$, where $D$ is the population density and $\sigma^{2}$ is the intergenerational variance in spatial location (i.e. dispersal distance). This yields an estimate of $4 \mathrm{D} \pi \sigma^{2} \approx 125$ individuals. Mathis (1991) estimated that the density near our study site as 2.82 individuals $/ \mathrm{m}^{2}$, giving an estimate of 3.53 for $\sigma^{2}$. However, densities on our study site may be somewhat lower than those at Mathis' site and effective population size is likely to be less than absolute population size. Therefore, we also consider that the effective population size may be half or even one-quarter of the Mathis (1991) estimate. These assumptions yield values for $\sigma^{2}$ of 7.05 and 14.11, respectively.

\section{Sex-bias in dispersal}

Neither our sex-based comparisons of $R$ nor AIc provide evidence of sex-biased dispersal. Mean pair-wise $R$ values for males $(0.23)$ and females $(0.21)$ were nearly identical $(T=0.48, P=0.95)$. Similarly, the mean AIc of the 131 males $(0.247)$ and the 109 females $(-0.297)$ were not significantly different $(P=0.137)$. We note that the high values of $R$ simply reflect the levels of differentiation across the transect and do not imply that individuals within a plot are necessarily close relatives (Queller and Goodnight, 1989).

\section{Discussion}

Analysis of microsatellite data from red-backed salamanders suggests a continuously distributed population with limited dispersal, resulting in an isolation-bydistance model of population structure. There was a clear signal of slight but detectable population structure from the Bayesian analysis of population structure, the assignment tests and the estimates of $F_{\mathrm{ST}}$. The Bayesian admixture model recovered seven of the eight plots, and the assignment tests demonstrated clear genetic differences among plots that increased with distance. Standard measures for population differentiation were small $\left(F_{\mathrm{ST}}\right.$ 0.01 to 0.06 ) but statistically significant even for some populations separated by as little as $200 \mathrm{~m}$. Hedrick
(1999; 2005) has demonstrated that standard fixation indices (e.g. $G_{\mathrm{ST}}$ ) are limited in magnitude by the amount of within-population homozygosity that is present in a given data set, so these estimates may be conservative. Although this traditional approach to analyzing population structure has many problems, in our analysis similar conclusions were reached using three different analytical approaches.

The Mantel test, assignment tests and Bayesian cluster analysis of individuals were all consistent with an isolation-by-distance model for population structure. Extrapolations of the second moment of dispersal distance from $F_{\mathrm{ST}}$ values (Rousset, 1997) suggested mean intergenerational dispersal of less than $20 \mathrm{~m}$. Similarly, Bayesian estimates of dispersal frequencies suggest the equivalent of a relatively small number of migrants (about six) per generation for plots separated by 200$300 \mathrm{~m}$. Although estimating dispersal rates from patterns of genetic differentiation is notoriously difficult (see Whitlock and McCauley (1999) and Neigel (2002) for a review), all our analyses were consistent in their suggestion of limited dispersal and isolation-by-distance for red-backed salamanders within our study site.

We found little evidence for sex-biased dispersal, despite the use of multiple statistical methods and relatively large sample sizes (six loci for approximately 400 individuals). The few other genetic studies of sexbiased dispersal in amphibians suggest female-biased dispersal in some species (Austin et al., 2003; Palo et al., 2004), and male-biased dispersal in others (Lampert et al., 2003). Mark-recapture data on sex-biased dispersal also indicate substantial variation among amphibian species (Marsh and Trenham, 2001). Our study suggests that redbacked salamanders may have little sex-biased dispersal, which is perhaps not surprising considering that both sexes are often territorial in this species (Mathis, 1991). However, given that dispersal appears limited for both sexes but no evidence for inbreeding (e.g. lower than expected heterozygosities) was found, it appears that red-backed salamanders may have some mechanism for inbreeding avoidance. One possibility is that these salamanders possess a kin recognition mechanism used in mate selection. Although kin recognition has not been demonstrated in Plethodon, it is known from several other amphibian taxa (Walls and Roudebush, 1991). In addition, red-backed salamanders are known to be able to distinguish neighbors from unknown individuals by smell (Jaeger, 1981) and males from females by fecal pellets alone (Mathis, 1990). Thus, kin recognition is at least a plausible mechanism for inbreeding avoidance in red-backed salamanders.

Amphibians with direct-development (i.e., no aquatic larval phase) have greatly diversified across North America, South America and Asia, and may account for $20 \%$ of all amphibian species. Driscoll (1998) studied a species of Australian frog of the genus Geocrinia with direct development, and showed extremely high levels of genetic differentiation on the scale of a few kilometers. Using allozymes, Driscoll observed $F_{\mathrm{ST}}$ values in the range of $0.30-0.69$ and genetic neighborhoods as small as $30 \mathrm{~m}$. Crawford (2003) studied population structure of direct-developing Eleutherodactylus frogs, and also uncovered extreme values of population differentiation $\left(F_{\mathrm{ST}}\right.$ of $0.5-1.0$ for most population pairs), although these estimates were made from DNA sequence data, and the 
populations were much further apart (more than $30 \mathrm{~km}$ ). Dubois (2004) has suggested that 'family mortality' (the loss of whole clutches to predators) may increase the rate of genetic divergence in amphibians with direct development. Our data, which suggest population structure over very short distances, are certainly consistent with this prediction. Although he suggests that this may be tied directly to higher rates of speciation, our experimental design does not allow any direct speculation about this; however, the genus Plethodon is one of the most speciose genera of North American salamanders.

The more numerous studies of genetic structure in pond-breeding amphibians have found genetic differentiation to be less pronounced, but highly variable among species. Several previous studies have reported detectable genetic differentiation on spatial scales only somewhat larger than the scale of our study (Lampert et al., 2003; Palo et al., 2004). Other studies using microsatellites have found much less genetic structure on these small scales and have suggested that some amphibians may disperse farther than previously recognized (Newman and Squire, 2001; Burns et al., 2004). Thus, our study puts terrestrial salamanders near the low end of the range for gene flow in amphibians. If genetic structure is generally more pronounced in species with direct development, this could suggest that this lifehistory adaptation tends to lead to low dispersal and high species diversity; alternatively, this is also consistent with Dubois' (2004) prediction that direct-developing amphibians may show more pronounced population structure owing to whole-clutch mortality.

Our interpretation of the genetic data for red-backed salamanders is consistent with mark-recapture data showing very limited dispersal for nearby populations (Mathis, 1991; Gillette, 2003). However, these data are more difficult to reconcile with red-backed salamanders' ability to colonize new habitats (Marsh et al., 2004), their long-distance homing abilities (Kleeberger and Werner, 1982; Marsh et al., 2004) and their substantial postPleistocene range expansion (Petranka, 1998). There are several possibilities that could explain this apparent discrepancy. The first is that dispersal is not necessarily the same as gene flow. Field studies have suggested the presence of a large proportion of non-territorial floaters who may lack the opportunity to breed (Mathis, 1991). Thus, young adults may move fairly long distances but fail to breed in areas that are already near carrying capacity (Jaeger, 1980). This scenario could account for the fact that the majority of colonists observed by Marsh et al. (2004) were newly mature adults, and could also explain how salamanders expand their range when suitable habitat becomes available. A related possibility is that dispersal behavior is flexible for individual salamanders. That is, salamanders may have the potential to disperse over a very large area, but use this ability only when suitable but unsaturated habitat is available. This could explain the homing ability of these animals, along with their colonization ability and potential for range expansion. Finally, it is possible that dispersal behavior varies throughout the range of red-backed salamanders. Salamanders in Virginia and near the core of the range may disperse minimally, as most available habitats have likely been colonized. Conversely, closer to range margins, salamanders may have a greater propensity for dispersal. Some variation in behavior is in fact known to exist across the range of red-backed salamanders. Territorial behavior in red-backed salamanders is well-documented from Virginia populations (Mathis, 1989, 1991), but appears to be absent from populations in Michigan (Quinn and Graves, 1999). Models and empirical results for other species suggest that dispersal may be selected for at range margins, leading to greater dispersal ability at margins relative to core areas (Simmons and Thomas, 2004). Thus, there are several potential explanations for the apparently contradictory picture of red-backed salamander dispersal, and each of these possibilities merits further investigation.

\section{Acknowledgements}

We thank Graham Milam, Noelle Beckman and Nick Gorham for help with collecting samples. Henry Wilbur provided advice on site selection and Jesse Overcash gave permission to use the research site. Collection of samples was covered by Virginia Department of Game and Inland Fisheries permit 21066 and funding was provided by NSF DEB-0235695 to DMM and PRC.

\section{References}

Austin JD, Davila JA, Lougheed SC, Boag PT (2003). Genetic evidence for female-biased dispersal in the bullfrog, Rana catesbeiana (Ranidae). Mol Ecol 12: 3165-3172.

Avise JC, Hamrick JL (1996). Conservation Genetics: Case Histories from Nature. Chapman \& Hall: New York, NY.

Balloux F, Goudet F (2002). Statistical properties of population differentiation estimators under stepwise mutation in a finite island model. Mol Ecol 11: 771-783.

Beerli P (2004). MIGRATE, Version 2.0: Migrate Documentation. http:/ / popgen.csit.fsu.edu/migrate.download.html.

Beerli P (2006). Comparison of Bayesian and maximumlikelihood inference of population genetic parameters. Bioinformatics 22: 341-345.

Beerli P, Felsenstein J (1999). Maximum-likelihood estimation of migration rates and effective population numbers in two populations using a coalescent approach. Genetics 152: 763-773.

Beerli P, Felsenstein J (2001). Maximum likelihood estimation of a migration matrix and effective population sizes in $n$ subpopulations by using a coalescent approach. Proc Natl Acad Sci USA 98: 4563-4568.

Bonnet E, Van de Peer Y (2002). ZT: a software tool for simple and partial Mantel tests. J Stat Software 7: 10.

Burns EL, Eldridge MDB, Houlden BA (2004). Microsatellite variation and population structure in a declining Australian Hylid Litoria aurea. Mol Ecol 13: 1745-1757.

Connors LM, Cabe PR (2003). Isolation of dinucleotide microsatellite loci from red-backed salamanders (Plethodon cinereus). Mol Ecol Notes 3: 131-133.

Corander J, Waldmann P, Sillanpää MJ (2003). Bayesian analysis of genetic differentiation between populations. Genetics 163 367-374.

Corander J, Waldmann P, Marttinen P, Sillanpää MJ (2004). BAPS2: enhanced possibilities for the analysis of genetic population structure. Bioinformatics 20: 2363-2369.

Crawford AJ (2003). Huge populations and old species of Costa Rican and Panamanian dirt frogs inferred from mitochondrial and nuclear gene sequences. Mol Ecol 12: 2525-2540.

Dieringer D, Schlötterer C (2003). Microsatellite analyser (MSA): a platform independent analysis tool for large microsatellite data sets. Mol Ecol Notes 3: 67-169.

Driscoll DA (1998). Genetic structure of the frogs Geocrinia lutea and Geocrinia rosea reflects extreme population divergence 
and range changes, not dispersal barriers. Evolution 52 : $1147-1157$.

Dubois A (2004). Developmental pathway, speciation and supraspecific taxonomy in amphibians 1 . Why are there so many frog species in Sri Lanka? Alytes 22: 19-37.

Excoffier L, Smouse PE, Quattro JM (1992). Analysis of molecular variance inferred from metric distances among DNA haplotypes: application to human mitochondrial DNA restriction data. Genetics 131: 479-491.

Funk WC, Blouin MS, Corn PS, Maxell BA, Pilliod DS, Amish S et al. (2005). Population structure of Columbia spotted frogs (Rana luteiventris) is strongly affected by the landscape. Mol Ecol 14: 483-496.

Garza JC, Williamson EG (2001). Detection in population size using data from microsatellite loci. Mol Ecol 10: 305-318.

Gibbs JP (1998). Genetic structure of redback salamander Plethodon cinereus populations in continuous and fragmented forests. Biol Conserv 86: 77-81.

Gillette JR (2003). Population ecology, social behavior, and intersexual differences in a natural population of red-backed salamanders: a long-term field study. $\mathrm{PhD}$ dissertation, University of Louisiana.

Gillette JR, Peterson MG (2001). The benefits of transparency: candling as a simple method for determining sex in redbacked salamanders (Plethodon cinereus). Herpetol Rev 32: 233-235.

Goudet J (2001). FSTAT, a program to estimate and test gene diversities and fixation indices (version 29.3). http:// www.unil.ch/izea/softwares/fstat.html.

Hedrick PW (1999). Perspective: highly variable loci and their interpretation in evolution and conservation. Evolution 53: 313-318.

Hedrick PW (2005). A standardized genetic differentiation measure. Evolution 59: 1633-1638.

Highton R (1977). Comparisons of microgeographic variation in morphological and electrophoretic traits. Evol Biol 10: 397-436.

Highton R, Webster TP (1976). Geographic protein variation and divergence in populations of the salamander Plethodon cinereus. Evolution 30: 33-45.

Jaeger RG (1980). Density-dependent and density-independent causes of extinction of a salamander population. Evolution 34: 617-621.

Jaeger RG (1981). Dear enemy recognition and the costs of aggression between salamanders. Am Nat 117: 962-974.

Kleeberger SR, Werner JK (1982). Home range and homing behavior of Plethodon cinereus in Northern Michigan. Copeia 1982: 409-415.

Knapp SM, Haas CA, Harpole DN, Kirkpatrick RL (2003). Initial effects of clearcutting and alternative silvicultural practices on terrestrial salamander abundance. Conserv Biol 17: $752-762$.

Lampert KP, Rand AS, Mueller UG, Ryan MJ (2003). Fine-scale genetic pattern and evidence for sex-biased dispersal in the tungara frog, Physalaemus pustulosus. Mol Ecol 12: 3325-3334

Marsh DM, Thakur KA, Bulka KC, Clarke LB (2004). Dispersal and colonization through open fields by a terrestrial, woodland salamander. Ecology 85: 3396-3405.
Marsh DM, Trenham PC (2001). Metapopulation dynamics and amphibian conservation. Conserv Biol 15: 40-49.

Marvin GA (1998). Territorial behavior of the plethodontid salamander Plethodon kentucki: influence of habitat structure and population density. Oecologia 114: 133-144.

Mathis A (1989). Do seasonal spatial patterns in a terrestrial salamander reflect reproductive behavior or territoriality? Copeia 1989: 788-791.

Mathis A (1990). Territorial salamanders assess sexual and competitive information via chemical signals. Anim Behav 40: 953-962.

Mathis A (1991). Territories of male and female terrestrial salamanders: costs, benefits, and intersexual spatial associations. Oecologia 86: 433-440.

Neigel JE (2002). Is $F_{\mathrm{ST}}$ obsolete? Conserv Genet 3: 167-173.

Newman RA, Squire T (2001). Microsatellite variation and finescale population structure in the wood frog (Rana sylvatica). Mol Ecol 10: 1087-1100.

Palo JU, Lesbarreres D, Schmeller DS, Primmer CR, Merila J (2004). Microsatellite marker data suggest sex-biased dispersal in the common frog Rana temporaria. Mol Ecol 13: 2865-2869.

Petranka JW (1998). Salamanders of the United States and Canada. Smithsonian Institution Press: Washington, DC, USA.

Queller DC, Goodnight KF (1989). Estimating relatedness using genetic markers. Evolution 43: 258-275.

Quinn VS, Graves BM (1999). Space use in response to conspecifics by the red-backed salamander (Plethodon cinereus, Plethodontidae, Caudata). Ethology 105: 993-1002.

Raymond M, Rousset F (1995). Genepop: population genetics software for exact tests and ecumenicism. I Hered 86: 248-249.

Rousset F (1997). Genetic differentiation and estimation of gene flow from $F$-statistics under isolation by distance. Genetics 145: 1219-1228.

Schneider J, Roessli D, Excoffier L (2000). ARLEQUIN, Version 2.000: A Software for Population Genetics Data Analysis. lgb.uige.ch/arlequin/software.

Shaffer G, Fellers GM, Magee A, Voss R (2000). The genetics of amphibian declines: population substructure and molecular differentiation in the Yosemite Toad, Bufo canorus (Anura, Bufonidae) based on single-strand conformation polymorphism analysis (SSCP) and mitochondrial DNA sequence data. Mol Ecol 9: 245-257.

Simmons AD, Thomas CD (2004). Changes in dispersal during species' range expansions. Am Nat 164: 378-395.

Sites JW, Morando M, Highton R, Huber F, Jung RE (2004). Phylogenetic relationships of the endangered Shenandoah Salamander (Plethodon shenandoah) and other salamanders of the Plethodon cinereus group (Caudata: Plethodontidae). J Herpetol 38: 96-105.

Walls SC, Roudebush RE (1991). Reduced aggression toward siblings as evidence of kin recognition in cannibalistic salamanders. Am Nat 138: 1027-1038.

Weir BS (1990). Genetic Data Analysis: Methods for Discrete Population Genetic Data. Sinauer Associates: Sunderland, MA, USA.

Whitlock MC, McCauley DE (1999). Indirect measures of gene flow and migration: $F_{\mathrm{ST}} \neq 1 /(4 \mathrm{Nm}+1)$. Heredity 82: 117-125. 